\title{
Vision-based drowsiness detector for Real Driving Conditions
}

\author{
I. García, S. Bronte, L. M. Bergasa, J. Almazán, J. Yebes
}

\begin{abstract}
This paper presents a non-intrusive approach for drowsiness detection, based on computer vision. It is installed in a car and it is able to work under real operation conditions. An IR camera is placed in front of the driver, in the dashboard, in order to detect his face and obtain drowsiness clues from their eyes closure. It works in a robust and automatic way, without prior calibration. The presented system is composed of 3 stages. The first one is pre-processing, which includes face and eye detection and normalization. The second stage performs pupil position detection and characterization, combining it with an adaptive lighting filtering to make the system capable of dealing with outdoor illumination conditions. The final stage computes PERCLOS from eyes closure information. In order to evaluate this system, an outdoor database was generated, consisting of several experiments carried out during more than 25 driving hours. A study about the performance of this proposal, showing results from this testbench, is presented.
\end{abstract}

Index Terms-Intelligent Transportation Systems, Driver Drowsiness, PERcentage of eye CLOSure (PERCLOS), Illumination independence.

\section{INTRODUCTION}

Sleepiness during driving has been proven to be one of the main causes of traffic accidents. Specifically, Klauer et al. [1] has shown that driving when the user is sleepy increases the risk of having an accident between four and six times, compared to driving when the user is awake. Furthermore, the risk of suffering an accident is higher at night [2] or in situations with sleep deprivation [3]. In fact, at least 15-20\% of all vehicle accidents have been estimated to be sleepiness related [4]. Therefore, it is convenient to develop a system which monitors the physical and mental state of the driver. It should alert at the critical moment when the driver is getting fatigued, preventing accidents.

In the last decade, diverse techniques have been developed for monitoring drivers. The techniques used to detect driver's sleepiness can be generally divided into three main categories [5]. The first category includes methods based on biomedical signals [6] and [7]. Usually, they require electrodes attached to the body, which often causes annoyance to the driver. Most of them are far from being introduced in the market [8].

The second category includes methods based on driving behaviour. They basically evaluate variations in several signals recorded by CAN bus [9], [10], which are easy to acquire. That is the reason why some of these systems have entered the commercial market [11], [12] and [13] but there are few details available about them to perform a proper

I. García, S. Bronte, L. M. Bergasa, J. Almazán and J. Yebes are with the Electronics Department, Polytechnic School, University of Alcalá, Madrid, Spain. email: ivan.garcia, sebastian.bronte, bergasa, javier.almazan, javier.yebes@depeca.uah.es. comparison. They are subjected to constraints related to the kind of vehicle or driver, so these usually require long training periods.

The third category is based on visual asessment. Computer vision can be a natural and nonintrusive technique for monitoring driver's state from face images. These approaches are effective due to sleepiness is reflected through the face and eyes appearance. There are different kinds of algorithms proposed in the literature: methods based on standard cameras [14], [15]; IR cameras [5], [16] and [17]; and stereo cameras [16], [17]. Most of them pursuit to measure two different parameters: head pose and PERCLOS. Some of them are commercial, such as: Smart Eye [16] or Seeing Machines [17]. However, these products are still bound to environments under control and require hard calibration processes.

The validation for the experimental protocol is often conducted in laboratory, in driving simulators, for obvious safety reasons. Real road tests are more real but it implies higher risk [18]. There are not so many outdoor driving condition databases available in the literature. However, in order to validate the proposed system, it is important to have a balanced quantity of records of users in both scenarios.

This paper presents a non-intrusive approach for monitoring driver drowsiness, based on computer vision techniques, installed on a real car. An IR stereo camera is placed in front of the driver, in the dashboard, to obtain PERCLOS. Hence it has been shown to be the best parameter for outdoor conditions. The system is bound to real-time and robustness restrictions. Our proposal does not need a calibration process and includes techniques to overcome the typical problems of image processing algorithms, such as: changes of lighting conditions, user appearance and fast head movements. For the evaluation of the proposed system, several experiments have been carried out with a twofold objective: (a) To gather a database in outdoor driving conditions; and (b) to exhaustively validate the proposed system, using proper data.

In section II the simulator, the method used to study the drivers state and the generation of the ground truth are described. Section III depicts the algorithm used to detect drowsiness in drivers. After that, results associated with a video sequence and its comparison with the ground truth is presented in section IV. Finally in section V conclusions and future works are presented.

\section{EXPERIMENTS AND DATA COLLECTION}

\section{A. On board system}

Simulation aims to give the driver an opportunity to immerse himself in his habitual workplace and gives the researcher the opportunity to evaluate an early version of 
their algorithms. Naturalist simulation methodologies have been proven to be efficient in the ADAS design. In [19], a deep study in a naturalist simulator was performed prior to the study in a real car, in order to generate a proper testbench for real conditions. The on-board system was designed to be similar to the simulation one.

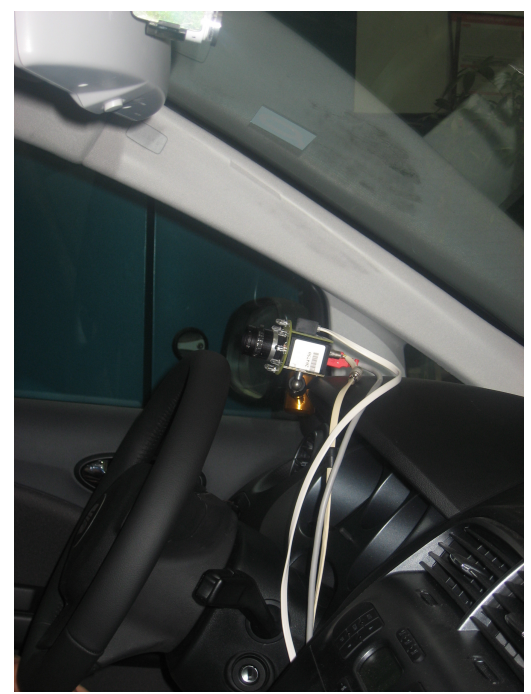

Fig. 1. On board camera installed in the car

For the real driving tests the chosen car was an Audi A4, equiped with an active IR illumination system for night conditions, a high resolution fireware camera pointing to the driver's face, and a computer for data acquisition (CAN bus and camera). A double pedal was installed in order to avoid security problems. A LDW was installed on the vehicle in order to evaluate some parameters that couldn't be acquired from the CAN bus.

The registered signals were the same as simulation. Speed and steering wheel angle were acquired directly from the CAN bus, which works at $50 \mathrm{~Hz}$. Lateral position and speed were acquired from the LDW system. The driver's face clues were registered using the visual acquisition system.

Using the previous experiences in the simulator, the psychologists used video data and vehicle signals to study driver's behaviour. As the car registers the same signals than in simulation, the study was performed in a similar way than in simulation. The ground truth was carried out using three experts that exhaustively evaluated the registered variables.

\section{B. Experimental protocol}

The main goal of the experimental tests is to recreate a suitable environment which would induce drowsiness in drivers during real driving operation. These tests are a continuation of the simulation exercises, that were created to develop and optimize the proposed algorithms [20].

The testbench was designed so that each user would carry out several driving sessions. They did not work before the tests for safety reasons. Drivers should not be too drowsy in order to avoid real car accidents. The tests were distributed in 3 sessions of 1 hour each, and a resting period of half hour between sessions. Every test session started at AP-2 road to complete $300 \mathrm{Km}$. It takes about 3 hours for each test. Main road features were: 9 meters width, 3.75 meters each lane and speed limit of $90 \mathrm{Km} / \mathrm{h}$.

Sleepiness detection is usually treated as a binary classification problem. Then, the performance is evaluated using a two level classificator, in which the output is updated every minute. It is based on the subjective sleepiness estimation from three experts. Taking the most voted estimation as ground truth, the target is classifying correctly as many driving periods as possible.

\section{AlgORITHM FOR VISUAL-BASED DROWSINESS DETECTION}

The system architecture flowchart is shown in Fig. 2. The input image, which is taken from the IR sensitive camera, corresponds to a driver's face, who can be affected by different states of drowsiness. The input image is processed to detect face and eyes applying Viola and Jones algorithm and a Kalman filter to track their position. Once the eyes are detected, iris centre location module is executed. It is based on integral projections. Then the eyes closure is calculated by using a Gaussian Model. From this parameter, PERCLOS is estimated [19].

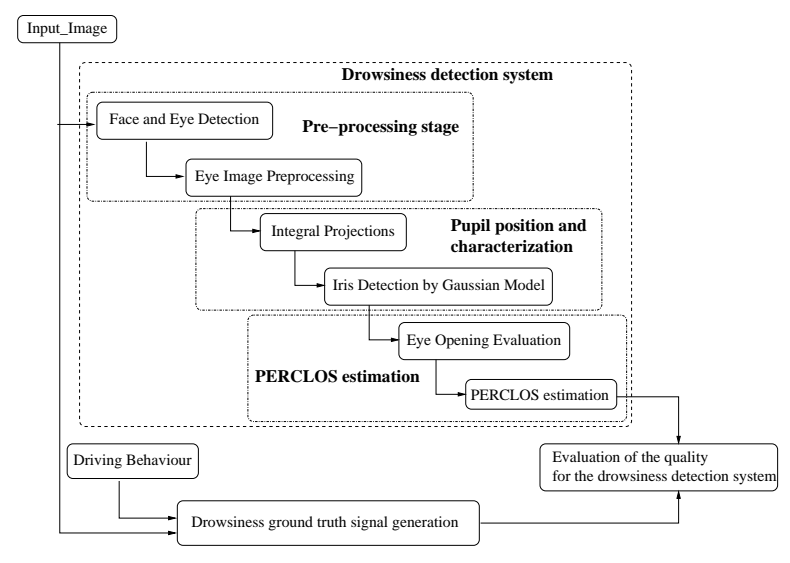

Fig. 2. General diagram

\section{A. Face and Eye detection}

In this step eyes position will be located throughout the whole image sequence. It is a very complicated challenge because of the different face colours, expressions, poses, relative sizes and very uneven illumination conditions.

Among all the techniques related to face and eye detection, Viola \& Jones [21] detector was selected, because face detection performance is high in frontal and few lateral face positions.

Eye search is carried out in two steps. The first one consists in looking for the face. As soon as the area is found, the eye detector is applied on it. Detection failures between consecutive images are smoothed using a Kalman tracker. It gives robustness to the PERCLOS measurement when dealing with fast face movements. It is easy to implement 
and its computational load is low, which is one of the restrictions of this system. The improvement using this filter is considerable, since it keeps the ROI enough stability to get good measurements.

Face and eyes' detection ROIs are shown in Fig. 3.

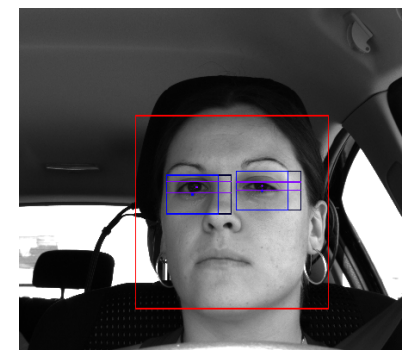

(a)

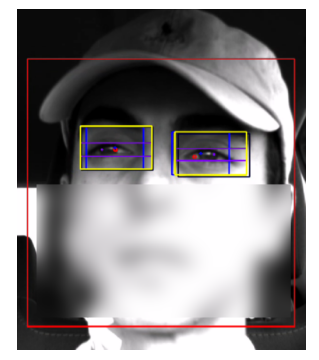

(b)
Fig. 3. Face and eyes detection in real conditions

\section{B. Eye Image Filtering}

Once the ROI is calculated for each eye, morphologic transformations are applied in order to remove bright artifacts. Therefore, a normalization to enhance gray levels is used. In Fig. 4 three images are shown. The first one is the original image, the second shows the result of a Hat filter [22], and the third shows the normalised image. A normalization is used instead of an equalization, since the latter has some side effects, like non-linear gray level distortion, illumination and shadow transformation, that are not convenient for this application.

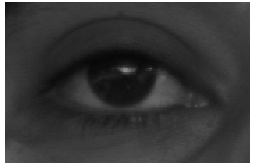

(a)

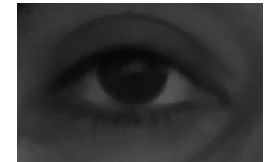

(b)

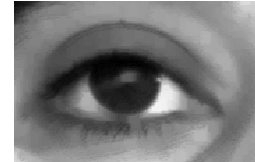

(c)
Fig. 4. (a) Original eye detection; (b) Hat transform; (c) Normalized image

This transformation depends on the illumination conditions, which is very challenging when dealing with outdoor conditions. Therefore, an adaptive algorithm is developed to get a better image in which evaluate the eye closure. This adaptive approach is composed of a sequence of filters. An example of this sequencial processing is shown in Fig. 5.

The applied masks are 5 pixels square, being their elements zero, except for the central row, which set to ones except for the central element. It increases its value from 1 to 6 , depending of the used filter. This filtering erases some of the artifacts, shadows and lighthings that can be found on the image. It takes only the main objects of the image, being most of the times, the iris and the upper eyelid.

The filters are sequencially applied until the eye closure evaluation falls below a certain threshold, which is experimentally set to 30 pixels. It is valid for all the tested users, although it is dependent of the kind of lens used and the distance between the face and the camera. The other stopping

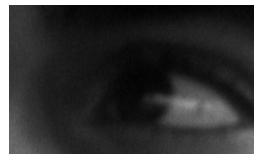

(a)

(d)

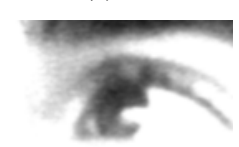

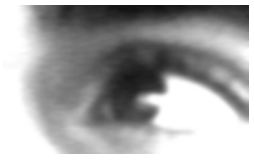

(b)

(e)

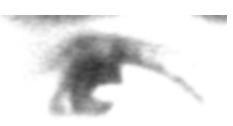

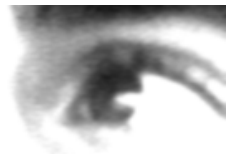

(c)

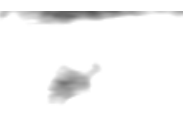

(f)
Fig. 5. (a) Input image; (b) 1st filter; (c) 2nd filter; (d)3rd filter; (e) 4th filter; (f) 5 th filter

criteria is the maximum number of applied filters, which is set to 6 . When any of the conditions are achieved, the current image is taken for the PERCLOS evaluation.

\section{Eye closure evaluation}

The eye closure evaluation algorithm is composed of integral projections, horizontal and vertical, from the detected eye area, to have an estimation of the iris centre.Eye closure, or the iris height, is evaluated measuring the standard deviation of the data and modeling them by a Gaussian (Fig. 6). From the empirical experience, the integral projections appeared to be Gaussian. The variance is estimated for both directions, the vertical one corresponds to the eye height, which is used to obtain the PERCLOS.

To evaluate the eye closure percentage, a previous calibration process is needed. The calibration process is automatically carried out during the first 10 seconds of the exercise. The driver has to look at the road, when he is awake, in a relaxed position and the algorithm calculates the nominal eye closure value.

Using this nominal value, the instantaneous percentage of eye closure is evaluated. It is computed from the ratio between the iris height in the current frame and its nominal value. The eye closure percentage is used in the next step, which evaluates PERCLOS.

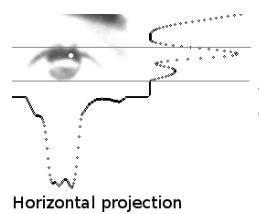

(a)

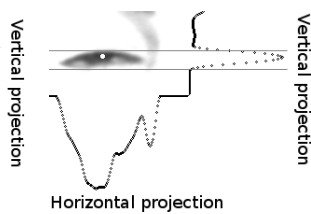

(b)

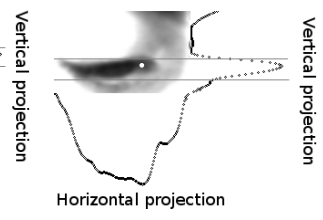

(c)
Fig. 6. Sample of aspect-ratio of the eye closure

When the driver's head is turned and the algorithm does not detect any iris the PERCLOS decreases due to the fact that it is a clue that the driver is awake.

\section{Drowsiness parameter estimation}

PERCLOS [23] is one of the most important parameters for driver drowsiness detection. It shows how much and how 
long the eyes are opened computing the average in a temporal window, whose duration is commonly accepted to be 20 secs.

To make this measurement more accurate, it is necessary to distinguish between blink and PERCLOS. Blinking time has to be removed from the estimation. Certain commonly accepted thresholds are already defined for eye closure, to introduce the measurements in the accumulators needed to estimate this parameter without blinking.

\section{EXPERIMENTAL RESULTS}

The testbench has the following characteristics: 10 different users with a total of 30 driving hours, 1296 awake minutes and 504 fatigue minutes. Drowsiness was detected from driving and driver-related signals [24].

The ground truth was composed by 2 states, awake and fatigued, trying to avoid the drowsy state, due to the risk for the driver.

The results obtained for 3 different users will be shown throughout this section. The first user is related to the simulation scenario, and the other two are related to real experiments.

Fig. 7 depicts 35 minutes for the first experiment. Some sample frames of an increasing PERCLOS are displayed in Fig. 8. Eye closure for the first minutes is depicted in Fig. 8.a and 8.b, corresponding to the awake state. As time goes by, eyes are significantly more closed, as it can be noticed in Fig. 8.d - 8.h, corresponding to a drowsy state.
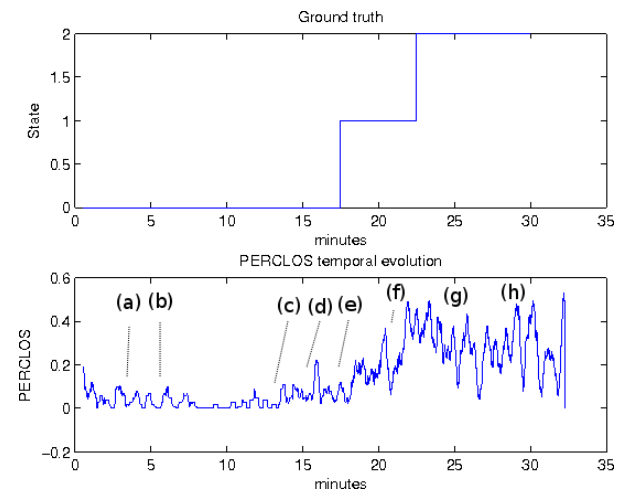

Fig. 7. Temporal evolution of PERCLOS for the drowsy state test

Hereafter, PERCLOS relation with the drowsiness is evaluated for real driving conditions in the following cases: 2 experiments with an awake driver and high illumination variations and one more experiment for a driver with fatigue symptoms.

From different condition tests, 2 sessions, for two users, with fatigue symptoms are shown in this paper. PERCLOS evolution for the second experiment is shown in Fig. 9. Fatigue appears between minute 110 and 140, as it is depicted in the sequence from Fig. 10.d - 10.g. In these images PERCLOS is higher than images Fig. 10.a and 10.b. In Fig. 10.h, the driver appears to be awake again, corresponding to the final of the test.

Hereafter, three sequences are displayed from the third experiment, corresponding to head rotation, dazzle caused

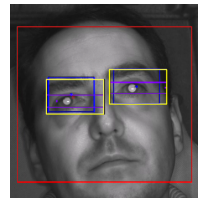

(a) $\mathrm{f}-5874$

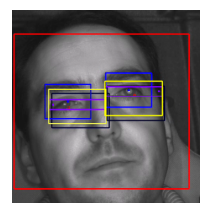

(e) f-30102

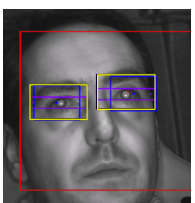

(b) $\mathrm{f}-8559$

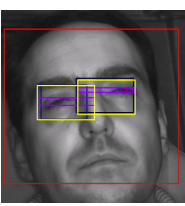

(f) $f-32249$

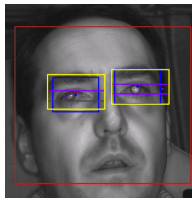

(c) f-27509

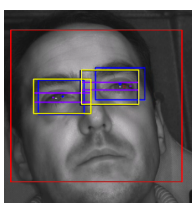

(g) f-39407

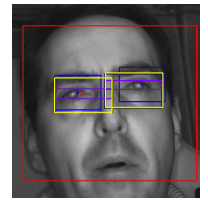

(d) $\mathrm{f}-27647$

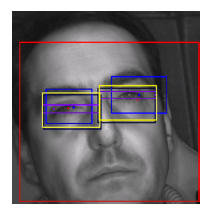

(h) f-39944
Fig. 8. Image sequence for the drowsy state test
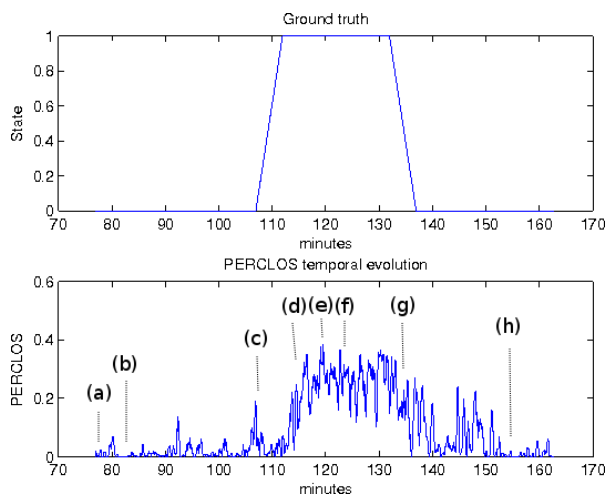

Fig. 9. PERCLOS temporal evolution for a real experiment

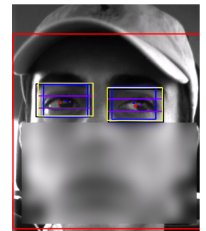

(a) $\mathrm{f}-8547$

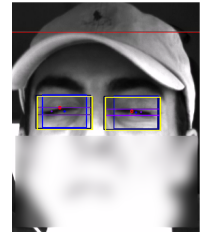

(e) $f-77130$

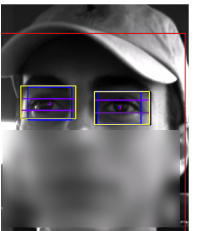

(b) f-17953

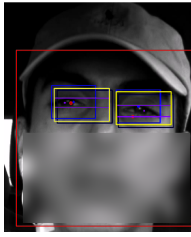

(f) $\mathrm{f}-82254$

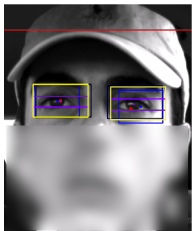

(c) f-62066

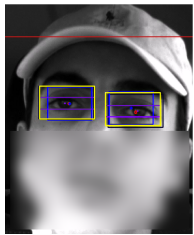

(g) f-106470

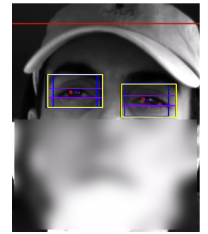

(d) f-74199

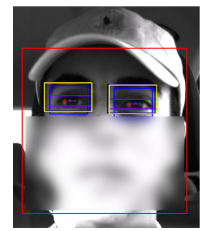

(h) f-124799
Fig. 10. Real test image sequence

by the sun, and going through a tunnel cases, in order to show the system behaviour in the worst working conditions.

For the first sequence, eye closure measurement when the user turns her head is depicted, getting an appropiated value for PERCLOS, that reflects the driver's state. In the first images, Fig. 11.a - 11.c, the driver begins turning slightly her head, keeping it turned between Fig. 11.d - 11.g, and getting back to the front in Fig. 11.h. In the whole sequence, PERCLOS estimation was correct. In Fig. 11.e an example 
of right eye detection error is shown.

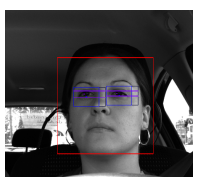

(a)

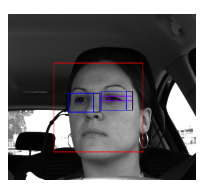

(e) f-11176

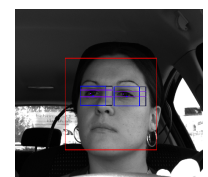

(b) f-11131

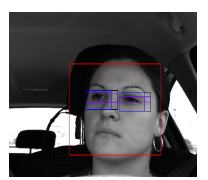

(f) $\mathrm{f}-11200$

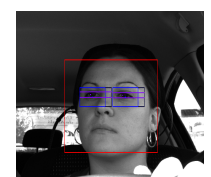

(c) f-11136

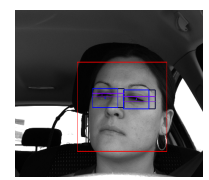

(g) f-11213

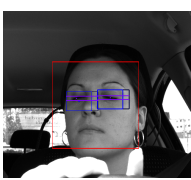

(d) f-11155

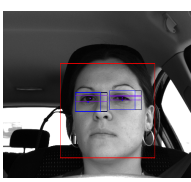

(h) f-11286
Fig. 11. Eye closure characterization when turning head

On the second sequence, depicted in Fig. 12, PERCLOS is evaluated with extreme sunlight conditions. Therefore, robustness can be verified in the transition among images 12.b and 12.e, where a great illumination change can be noticed. As it can be seen, eye detection is correct.

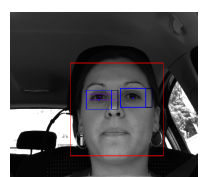

(a) $\mathrm{f}-12158$

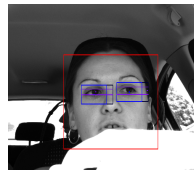

(e) f-12227

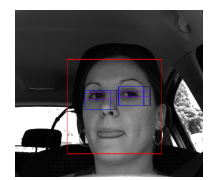

(b) f-12170

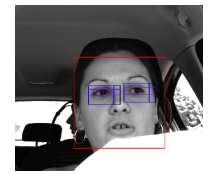

(f) $f-12228$

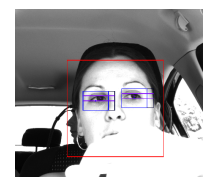

(c) f-12225

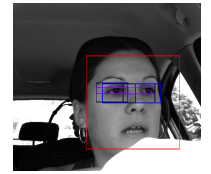

(g) f-12235

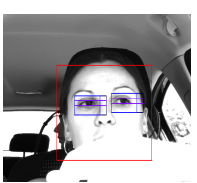

(d) $\mathrm{f}-12226$

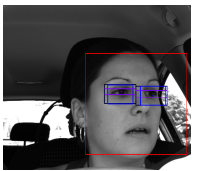

(h) f-12239
Fig. 12. Eye characterization under high sunlight lighting

The third sequence, depicted in Fig. 13, shows an example of driving through a tunnel. Images are getting dark and getting lighter. Some of them are almost black images (Fig. 13.a-b). In spite of this, eyes detection is quite good.

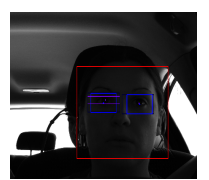

(a) f-13004

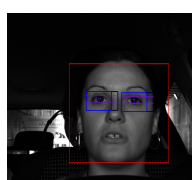

(e) f-13123

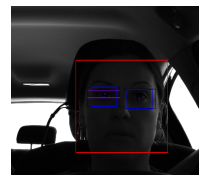

(b) f-13006

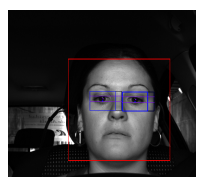

(f) $\mathrm{f}-13136$

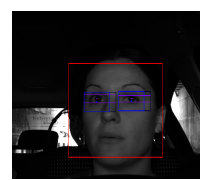

(c) $\mathrm{f}-13112$

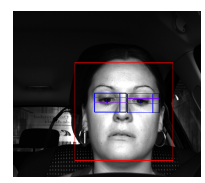

(g) f-13146

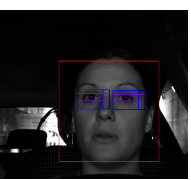

(d) $\mathrm{f}-13116$

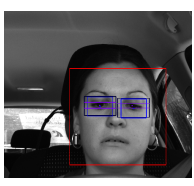

(h) f-13185
Fig. 13. Eye characterization while driving through a tunnel

PERCLOS evolution for this user is depicted in Fig. 14 and corresponds to 6-8 minutes interval.

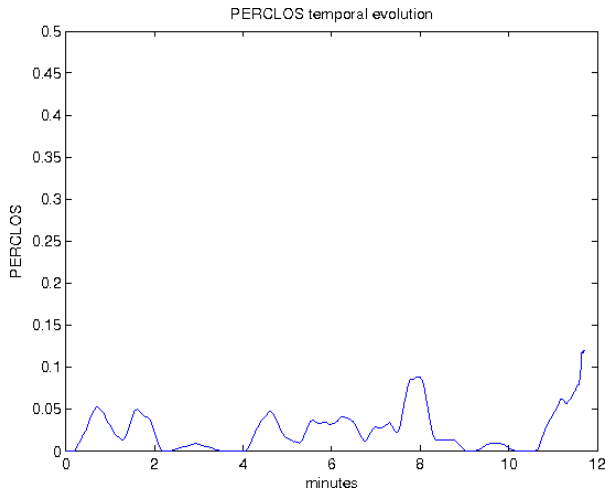

Fig. 14. PERCLOS temporal evolution in other real essay

Finally, the average specificity reached for this system, when the PERCLOS is compared against the ground truth, is $92.21 \%$, which represents quite good results, similar to the ones obtained in simulation [19]. In addition to that, the average sensitivity achieved is $79.94 \%$, which is a good value, taking into account that most of the commercial systems have a high false positive rate.

However, due to the complexity and the non-linearity of the human behaviour, some dependences between driverrelated and driving-related variables are established. These dependencies have been explored using a fusion using Neural Networks. Using an ANN (Artificial Neural Network) is possible to fuse both types of indicators to improve the recall rate. A deeper explanation of the fusion methodology is out of the scope of this paper. The average results for all the users are shown in table I.

TABLE I

DRIVER AND DRIVING INDICATOR COMBINATION

\begin{tabular}{l|ccc}
\hline \hline Indicators & Specificity & Sensibility & avg value \\
\hline \hline PERCLOS-MSE_lateral_position & 95.83 & 85.54 & 90.68 \\
\hline PERCLOS-Lateral_position & 92.32 & 85.00 & 88.66 \\
\hline PERCLOS-Wheel_movement & 90.54 & 82.50 & 86.52 \\
\hline \hline PERCLOS & 92.21 & 79.94 & 86.07 \\
\hline \hline
\end{tabular}

High recall and low false rates makes the system good to be implemented in a real car, since the user annoyance, due to false positives, is quite low, and recall rate assures that the drowsiness estimation is correct most of the time.

\section{CONCLUSIONS AND FUTURE WORKS}

This paper presents a non-intrusive approach for monitoring driver drowsiness, based on computer vision techniques, installed on a real car, capable of dealing with real operation conditions. Most of the previously addressed works can only deal with indoor conditions, like naturalistic simulators or night conditions, in which the illumination is under control.

The implemented system is user-independent and can evaluate PERCLOS indicator in real time, in both, simulation and real operation scenarios. Results obtained with our system are similar or even better than other commercial ones [17], [16], being more flexible and opensource. The commercial 
systems often require a non-trivial calibration procedure, to adjust the detection.

The proposed PERCLOS estimation method has demonstrated to be accurate, showing specificity of $92.21 \%$ and a sensitivity of $79.84 \%$ in average, considering only this parameter. The fusion of this indicator with other driving indicators improves the results, reaching a recall rate of $90.68 \%$.

The computer vision algorithms, do not need a calibration set up to determine the eyes closure. However, PERCLOS assessment needs a nominal eye closure value. This nominal value is dependent on the constraints of the driver's eye and the selected camera lens, although it is estimated in an initial automatic process, which makes this system flexible to be installed in any vehicle.

The developed system is robust against strong illumination variations, and complies real time computation requirements. This system has been widely tested, not only for outdoor conditions, but for controlled illumination conditions in a naturalistic simulator [19].

Generating a ground truth for drowsiness driver signal by psychologists experts is the key to correctly evaluate the recall rate of any method related to drowsiness detection.

The application of appearance-based techniques by using SVM classifiers could complement the characterization algorithms implemented, in order to improve the eye aperture estimation. In addition, SVM classifier could be used to improve eye blinking detection.

The data fusion with other indicators like blink frequency, fixed gaze, lane deviation and amplitude of wheel turns, could improve the performance of the system.

\section{ACKNOWLEDGMENTS}

This work has been funded in part by the Economy and Competitibity through the project ADD-Gaze(TRA201129001-004-01), as well as by the multinational company FICOSA throught MARTA. Authors want to thank the work of all the drivers that collaborated in the tests. Thanks to the experts that helped us elaborating the ground truth signal from the video sequences.

\section{REFERENCES}

[1] S. G. Klauer, T. A. Dingus, V. L. Neale, , and J. D. Sudweeks, "The impact of driver inattention on near-crash/crash risk: An analysis using the 100-car naturalistic driving study data," National Highway Traffic Safety Administration, DC, DOT HS, vol. 810, 2006.

[2] T. Akerstedt, G. Kecklund, and L. Hörte, "Night driving, season, and the risk of highway accidents." Slee, vol. 24, pp. 401-406, 2001.

[3] J. Connor, R. Norton, S. Ameratunga, E. Robinson, I. Civil, R. Dunn, J. Bailey, and R. Jackson, "Driver sleepiness and risk of serious injury to car occupants: Population based control study." British Medical Journal, vol. 324, pp. 1125-1129, 2002.

[4] J. Horne and L. Reyner, "Vehicle accidents related to sleep: A review." Occupational and Environmental Medicine, vol. 56, pp. 189-294, 1999.

[5] L. M. Bergasa, J. Nuevo, M. Á. Sotelo, R. Barea, and M. E. L. Guillén, "Real-time system for monitoring driver vigilance," IEEE Transactions on Intelligent Transportation Systems, vol. 7, no. 1, pp. 63-77, 2006.

[6] C. Papadelis, Z. Chen, C. Kourtidou-Papadeli, P. Bamidis, I. Chouvarda, E. Bekiaris, and N. Maglaveras, "Monitoring sleepiness with on-board electrophysiological recordings for preventing sleep-deprived traffic accidents." Clinical Neurophysiology, vol. 118, no. 9, pp. 19061922, September 2007.
[7] J. Faber, "Detection of different levels of vigilance by eeg pseudo spectra." Neural Network World, vol. 14, no. 3-4, pp. 285-290, 2004.

[8] N. Wright, B. Stone, T. Horberry, and N. Reed, "A review of in-vehicle sleepiness detection devices," TRL Limited, Published Project Report PPR157, 2007.

[9] T. Wakita, K. Ozawa, C. Miyajima, K. Igarashi, K. Itou, K. Takeda, and F. Itakura, "Driver identification using driving behavior signals," IEICE - Trans. Inf. Syst., vol. E89-D, no. 3, pp. 1188-1194, 2006.

[10] Y. Takei and Y. Furukawa, "Estimate of driver's fatigue through steering motion." IEEE International Conference on Systems, Man and Cybernetics., vol. 2, p. 1765-1770, 2005.

[11] "Volvo car corporation," http://www.media.volvocars.com.

[12] "Mercedes-benz," http://www.emercedesbenz.com/Aug08.

[13] "Lexus," http://www.testdriven.co.uk/lexus-1s-600h.

[14] T. D’Orazio, M. Leo, C. Guaragnella, and A. Distante, "A visual approach for driver inattention detection," Pattern Recogn., vol. 40, no. 8, pp. 2341-2355, 2007.

[15] M. Suzuki, N. Yamamoto, O. Yamamoto, T. Nakano, and S. Yamamoto, "Measurement of driver's consciousness by image processing -a method for presuming driver's drowsiness by eye-blinks coping with individual differences." Systems, Man and Cybernetics, vol. 4, p. 2891-2896, 2006.

[16] "Smart eye," http://www.smarteye.se.

[17] "Seeingmachines," http://www.seeingmachines.com.

[18] J. Bos, W. Bles, and E. Groen, "A theory on visually induced motion sickness," Displays, vol. 29, no. 2, pp. 47-57, 2008

[19] I. García, S. Bronte, L. Bergasa, N. Hernández, B. Delgado, and M. Sevillano, "Vision-based drowsiness detector for a realistic driving simulator," in IEEE Intelligent Transportations Systems Conference (ITSC), 2010.

[20] N. Stanton, P. Salmon, G. Walter, and C. B. . D. Jenkins, "Human factors methods: A practical guide for engineering and design," 2005.

[21] P. Viola and M. J. Jones, "Robust real-time face detection," Int. J. Comput. Vision, vol. 57, no. 2, pp. 137-154, 2004.

[22] E. Dougherty, An Introduction to Morphological Image Processing, 1992.

[23] J. F. May and C. L. Baldwin, "Driver fatigue: The importance of identifying causal factors of fatigue when considering detection and countermeasure technologies," Transportation Research Part F: Traffic Psychology and Behaviour, vol. 12, no. 3, pp. 218 - 224, 2009. [Online]. Available: http://www.sciencedirect.com/science/article/B6VN84VFBYG1-1/2/07087f8c3b6f88f9e9ed6996388d01ed

[24] I. Daza, N. Hernandez, L. Bergasa, I. Parra, J. Yebes, M. Gavilan, R. Quintero, D. Llorca, and M. Sotelo, "Drowsiness monitoring based on driver and driving data fusion," in Intelligent Transportation Systems (ITSC), 2011 14th International IEEE Conference on, oct. 2011 , pp. $1199-1204$. 